\title{
Las mujeres y el envejecimiento en la investigación social (1950-2018)
}

\author{
Herminia Gonzálvez Torralbo' (iD) 0000-0002-4929-2521 \\ Menara Lube Guizardi ${ }^{2,3}$ (iD) 0000-0003-2670-9360 \\ 'Universidad Central de Chile, Vicerrectoria Académica y Facultad de Derecho y \\ Humanidades, Santiago de Chile, Chile.8370178-r-aguayo@ucentral.cl \\ ${ }^{2}$ Consejo Nacional de Investigaciones Científicas y Tecnológicas de Argentina, Buenos \\ Aires, Argentina \\ 3Universidad de Tarapacá, Arica, Chile. 1000000 - dipog@uta.cl
}

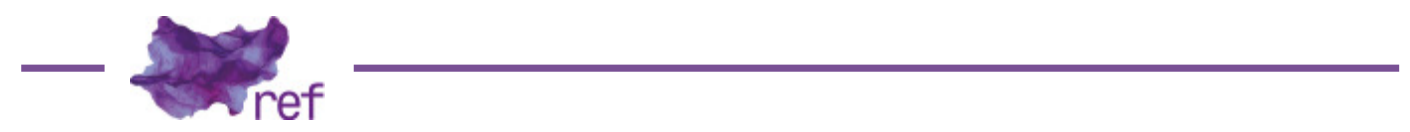

Resumen: Presentamos un estado del arte sobre los estudios del envejecimiento femenino en las ciencias sociales anglófonas e hispanohablantes, indagando cómo las mujeres mayores fueron representadas en estas investigaciones y buscando captar las contribuciones del feminismo a estos estudios. Revisaremos la emergencia de los debates gerontológicos sobre el envejecimiento (19501970) y la influencia de los posicionamientos feministas en los trabajos sociológicos y antropológicos sobre el tema (1970-1990). Discutiremos los giros hacia la confluencia entre género y edad en los estudios sociales (1990-2000) y la contribución crítica latinoamericana en castellano, articulada alrededor del concepto de organización social de los cuidados (2000-2010). En las consideraciones finales, ofreceremos nuestras reflexiones sobre la construcción de una perspectiva feminista en los estudios del envejecimiento femenino.

Palabras-clave: género; envejecimiento; feminismo; organización social de los cuidados

Women and aging in social research (1950-2018)

Abstract: The article presents a state of the art on the studies of female aging in the anglophone and Spanish speaker social sciences. Our review investigates how older women were represented in these investigations, seeking to capture the contributions of feminism to these studies. We will start by reviewing the emergence of gerontological debates on aging (1950-1970), and by analyzing the influence of feminist positions in sociological and anthropological works on the subject (1970-1990). We will also discuss the epistemological turns towards the confluence between gender and age in social studies (1990-2000), and the Spanish speaker Latin American critical contribution to this debate, articulated around the concept of social organization of care (2000-2010). In the final remarks, we will offer our reflections on the construction of a feminist perspective in the studies of female aging.

Keywords: Gender; Aging; Feminism; Social Organization of Care.

As mulheres e o envelhecimento na pesquisa social (1950-2018)

Resumo: Apresentamos um estado da arte sobre os estudos do envelhecimento feminino nas ciências sociais anglófonas e hispanofalantes, indagando como as mulheres idosas foram representadas nestas pesquisas e buscando captar as contribuições do feminismo a estes estudos. Começaremos revisando a emergência dos debates gerontológicos sobre o envelhecimento (1950-1970) e analisando a influência dos posicionamentos feministas nos trabalhos sociológicos e antropológicos sobre o tema (19701990). Discutiremos, ademais, os giros da confluência entre gênero e idade nos estudos sociais (19902000) e a contribuição crítica hispanofalante latino-americana ao debate, articulada em torno ao conceito de organização social dos cuidados (2000-2010). Nas considerações finais, oferecemos nossas reflexões sobre a construção de uma perspectiva feminista nos estudos sobre o envelhecimento feminino.

Palavras-chave: gênero; envelhecimento; feminismo; organização social dos cuidados. 


\section{Invisibles, dependientes y problemáticas}

Este artículo presenta un estado del arte de los estudios sobre el envejecimiento femenino en las ciencias sociales anglófonas e hispanohablantes a partir de la segunda mitad del siglo XX. Se trata de un campo de discusiones prolijo y diverso, involucrando varias disciplinas del conocimiento -gerontología, sociología, antropología, demografía y trabajo social-y cuya síntesis difícilmente podría limitarse a un único texto. Primero, en función de la enorme heterogeneidad de marcos analíticos, conceptualizaciones y conclusiones que se observa en los trabajos sobre el tema y que responde, a su vez, a la pluralidad de contextos y momentos históricos que enmarcan la producción de estas obras. Segundo, porque estos estudios suscitaron polémicas que exceden al mundo académico. Estas publicaciones sirvieron de orientación a las políticas públicas que moldearon, en las últimas cuatro décadas, las respuestas dadas por los Estados (y también su omisión) sobre los procesos de envejecimiento y sobre las demandas de cuidado de sus poblaciones.

Considerando estas características, optamos por desarrollar una revisión críticamente orientada de la literatura. La selección de los textos y debates que abordaremos se guía por dos objetivos: 1) analizar cómo las mujeres mayores fueron representadas y categorizadas en las ciencias sociales anglófonas e hispanohablantes desde 1950; 2) captar las contribuciones y aportaciones del feminismo a los estudios del envejecimiento, identificando aquellos campos de investigación que permanecen "al descubierto". Este recorte conduce a una revisión que, más allá de sus cualidades, tiene un alcance limitado: el presente texto no revisita la amplia discusión sobre el tema realizada desde los 40 s en francés, y también la diversa y contundente producción desarrollada por las ciencias sociales luso-hablantes, por citar tan solo dos ejemplos. Particularmente en el caso de las ciencias sociales producidas en Brasil, encontramos una prolija y vanguardista investigación, ya en los 70, de autoras como Myriam Lins de Barros (2006). A su contribución, se siguen los trabajos de Guita Debert (1999), Clarice Peixoto (2000), Cornélia Eckert (2002), que han constituido contribuciones tempranas a varios de los debates que hoy entendemos fundamentales para comprender la relación entre género, edad y desigualdades sociales. Si nos dedicamos a revisar la literatura hispanohablante y anglófona es, precisamente, con el intuito de ofrecer elementos para comparar las producciones en estos idiomas con aquellas elaboradas en otros. Sugerimos que este ejercicio es un paso inicial, y por lo mismo fundamental, a la hora de pensar cómo avanzar en la visibilización de estos temas en las ciencias sociales producidas en Latinoamérica (en todos los idiomas que representan la región).

Otro punto de reflexión que respalda nuestro ejercicio en el presente artículo refiere a la interpretación de los géneros como procesos relacionales, conflictivos y sumamente heterogéneos. Así, partimos de la premisa de que el envejecimiento constituye una experiencia diferencial para todas y cada una de las identidades genéricas. Las identidades femeninas experimentarán este proceso de forma particular; como también lo harán las demás identidades (masculinas, transexuales, bisexuales, gays, queers, entre otras). Particularmente en este artículo, nos centraremos en revisar aquellas investigaciones que han planteado el lugar de las mujeres heterosexuales en los procesos de vejez. Esto no significa que entendamos la identidad femenina como sinónimo de identidad genérica. Tampoco significa que asumamos la heteronormatividad de esta designación como elemento estructurante de una teorización sobre género y vejez. La razón que nos lleva a centrarnos en las experiencias femeninas se debe a un interés empírico puntual: el estudio de estado del arte que aquí presentamos enmarcó nuestros esfuerzos analíticos iniciales en un proyecto de investigación sobre las problemáticas particulares enfrentados por las mujeres heterosexuales en su proceso de envejecimiento en Chile. Nos centramos en ellas porque los diagnósticos sociales en aquel país apuntan a una generalización de procesos de marginación femenina en la edad adulta (empobrecimiento, sobrecarga de cuidados y laboral, experiencia de violencias). Así, si nuestro enfoque en este texto se dirige a las mujeres, más que a otras identidades de género, esto se debe también a nuestra necesidad de respaldar nuestras incursiones empíricas puntuales.

En las ciencias sociales, las aportaciones feministas sobre la vejez tardaron en aparecer y, cuando lo hicieron, siguieron ocupando un lugar marginal entre las pautas de investigación. Los estudios del envejecimiento desarrollados desde la gerontología contemplaban, ya desde 1940, algunas reflexiones sobre las diferencias entre las experiencias masculinas y femeninas (José YUNI; Claudio URBANO, 2008, p. 152-153). Pero es solo cinco décadas más tarde que observamos un giro epistémico que centraliza la dimensión femenina del fenómeno en las investigaciones sociales. Fueron autoras como Nancy Datan (1989) las que empezaron a mostrar su preocupación por las mujeres que envejecen, evidenciando que ellas eran una "mayoría silenciosa" en camino de convertirse en un "problema social" que podría ser prevenido si se hicieran esfuerzos en educación, investigación y políticas públicas. Los estudios desarrollados entre 1980 y 1990 constituyeron, así, una apertura fundamental hacia perspectivas más críticas.

Con todo, se observa la persistencia de tres limitaciones estructurantes en el debate sobre envejecimiento femenino en las ciencias sociales. Primero, las mujeres mayores, cuando no fueron vistas como problema social, fueron olvidadas o silenciadas incluso de aquellas reflexiones 
sociológicas, antropológicas, historiográficas y politológicas sobre situaciones sociales para las cuales ellas constituían actores importantes (Toni CALASANTI; Katheleen SLEVIN; Neal KING, 2006). Segundo, que cuando ellas emergieron como un tema de investigación relevante, las discusiones sobre diferencias de raza, etnicidad o clase social estuvieron ausentes. Tercero, las mujeres mayores fueron conceptualizadas como un "todo homogéneo" (Diane GIBSON, 1996, p. 435).

Para dar cuenta de estos desenlaces, los apartados que siguen reconstruirán cronológicamente la articulación de aquellos estudios que situaron, de forma explícita, a las mujeres mayores como sujetos de estudio. En el segundo apartado, revisaremos el periodo que va de 1950 a 1970, marcado por la emergencia de los primeros debates gerontológicos, los cuales des-invisibilizaron a las mujeres mayores, aunque reincidiendo en una representación que las encasillaba como sujetos "dependientes" o "demandantes". En el tercero, que cubre el periodo entre 1970 y 1990, revisamos el surgimiento de los debates sociológicos y antropológicos sobre el tema, marcado por la incipiente atención de investigadoras y pensadoras feministas, abordándose las mujeres como un "problema social". El cuarto acompaña la formulación de la gerontología crítica feminista, entre 1990 y 2000 , observando un giro epistemológico que centraliza el género y edad. El quinto aborda la emergencia de la contribución latinoamericana en castellano a través del concepto de organización social de los cuidados (2000-2018). Finalizamos ofreciendo nuestro posicionamiento sobre estos debates.

\section{De la invisibilidad a la dependencia (1950-1970)}

El estudio de la vejez -masculina y femenina- no despertó mucho interés de las disciplinas de las ciencias sociales hasta 1980. La emergencia de un campo de estudios vinculado al tema deviene de la estructuración, desde 1940, de la gerontología (Elvira ARQUIOLA, 1995, p.154). Esta, si bien nace vinculada a las ciencias biológicas y de la salud -en una rama de la medicina denominada geriatría'- se acercó progresivamente a los debates y perspectivas sociales, criticando así las bases positivistas (y biologizantes) de su surgimiento disciplinario (YUNI; URBANO, 2008, p. 153).

Según Arquiola (1995, p. 153), la gerontología propulsó, en los años 40, una concientización sobre la relación entre los modos y estilos de vida y la constitución de la salud o de las enfermedades que, en aquél entonces, se habían distendido entre la población en la tercera edad. Este debate fue muy conflictivo. Una parte de la tradición médica -la británica y la francesa- suponía que la gerontología sería un campo amplio, que abarcaría la geriatría como una subespecialidad. En Estados Unidos, la postura hegemónica fue la inversa: se preconizó a la gerontología como un subcampo de la geriatría lo que culminó, en este país, en la separación de estas disciplinas. Obsérvese, no obstante, que todos estos debates emergen inicialmente en los países del norte global, siendo preferentemente desarrollados en Europa y Norte América hasta fines del siglo XX (RAMOS-TORO, 2015, p. 71). Esto tiene consecuencias para los países del sur global: las reflexiones sobre la vejez tardaron mucho en incluir las casuísticas propias de estos países.

Es en Estados Unidos donde, entre 1940 y 1960, la gerontología emergente inaugurará una primera generación de categorías analíticas que vinculan la vejez a los debates sociológicos. Estas incluían "Ias teorías de la actividad, de la desvinculación, de la modernización y de la subcultura de la vejez" (YUNI; URBANO, 2008, p. 152). Entre 1950 y 1960, estos trabajos pasaron a indagar sobre la experiencia de envejecimiento en su vinculación con fenómenos como la pobreza, el abandono familiar o la exclusión social (OSORIO, 2006, p. 7). A partir de 1960, la "Teoría de la Desvinculación", desarrollada por Elaine Cummings y William Henry (1961), emerge como paradigma hegemónico sobre el envejecimiento en los debates gerontológicos-posición que detentaría por aproximadamente medio siglo (OSORIO, 2006, p. 7). Dicha teoría asume que la vejez está socialmente construida, en las sociedades urbanas y capitalistas del norte global, a través de una exclusión fundante. El proceso de "desvinculación" del mercado laboral vivido por los sujetos mayores (debido a la jubilación) les empujaría a una condición social periférica, determinando que su acceso a bienes de consumo, relaciones sociales y dinámicas colectivas esté permanentemente demarcado por su "no participación" en el mundo productivo.

Sobre los desenlaces de esta teoría, recuperamos tres aspectos. 1) Su aplicación en países de la periferia capitalista donde la jubilación no siempre ha sido un derecho "universalizado" fue poco plausible. El argumento tenía capacidades explicativas para el contexto del Estado de Bienestar social estadounidense de los 60 . También era aplicable a varios contextos europeos de la Guerra Fría, cuando se produjo una distensión del derecho de jubilación salvaguardado por el Estado en los países centrales y occidentales del continente. Pero en los países "en desarrollo", la experiencia de los adultos mayores distaba ( $y$ dista) mucho de permitirles dejar tácitamente el

'Especialidad médica cuyos orígenes remontan a la primera década del siglo XX (Mónica RAMOS-TORO, 2015, p.71). En sus inicios, la geriatría se ocupaba tanto de los problemas fisiológicos y patológicos de la vejez, como también de las dimensiones legales y de asistencia de la población mayor (ARQUIOLA, 1995, p.152). 
mercado productivo cuando llegan a la edad jubilatoria². 2) La idea de "desvinculación" de la vejez condujo a una visión claramente pesimista, que asocia el envejecimiento a una condición inexorable de declinación física y social, retratándose a las personas ancianas como subsumidas a cuadros permanentes de dependencia (Malcolm JOHNSON, 2001, p.54; OSORIO, 2006, p. 7). 3) El interés de las ciencias sociales sobre estos debates fue del todo tardío: las primeras revisiones críticas surgen desde la propia gerontología ${ }^{3}$. En un texto considerado fundacional para la gerontología crítica, Robert Butler (1969) denuncia que los argumentos e investigaciones sobre el envejecimiento estarían condicionados por una mirada ideologizante sobre el papel de las personas mayores en las sociedades urbanas. Acuña, entonces, el término 'Age-Ism' ('edadismo'), para referirse a los perjuicios sociales que conforman las miradas "científicas" (Anna FREIXAS, 2015, p.29).

Siguiendo a Butler, otros investigadores elaborarán desde la gerontología, en los 70, una segunda generación de teorías sobre la vejez. Así, "aparecieron la teoría de la continuidad, de la competencia social, del intercambio, del ciclo vital, de la estratificación de la edad y de la economía política del envejecimiento" (YUNI; URBANO, 2008, p. 153). La aportación feminista en la antropología y sociología sobre la vejez solo emergerían a fines de los 70. Entre 1960 y 1970, los análisis feministas se preocuparon por las inequidades que experimentaban las mujeres en comparación con los hombres (GIBSON, 1996, p. 434), abordando problemas relacionados con el trabajo doméstico, maternidad e inequidad de acceso al "espacio público". Desde allí, el estudio de la relación entre género y envejecimiento no fue muy prolija. Es solamente cuando Simone de Beauvoir (1983[1970]) publica "La vejez" que el debate feminista centraliza el tema. La obra tuvo un impacto fundamental en el pensamiento antropológico sobre el género en su relación con la construcción generacional de los grupos sociales, afirmando que la condición de mujer mayor constituye un hecho biológico y cultural (DE BEAUVOIR, 1983, p. 20). De Beauvoir (1983, p. 647) pone en prensa, además, la discusión sobre el impacto de la condición de clase en el proceso de envejecer. A partir de ahí, observamos una progresiva coincidencia de las preocupaciones sobre la vejez esbozadas por pensadoras e investigadoras feministas con el enfoque de los gerontólogos.

De este "encuentro", emerge el enfoque del "doble riesgo" (Neena CHAPPELL; Betty HAVENS, 1980). De acuerdo con esta discusión, las mujeres mayores constituyen una subjetividad definida por dos elementos estructurantes de marginación (John KNODEL; Mary OFSTEDAL, 2003). Esta perspectiva representa un claro avance para la observación y análisis de las particularidades del envejecimiento femenino. Pero reincide en el pesimismo de las lecturas previas. El debate sobre el "doble riesgo" estuvo centrado en enunciar la cuestión de la vejez considerándola como parte de los "problemas de las mujeres" (GIBSON, 1996, p. 435). El resultado fue una tendencia de opacar la heterogeneidad de las mujeres mayores mostrándolas como un grupo dependiente que tiene poco que ofrecer a la sociedad y mucho que demandar.

\section{Un "problema social" (1970-1990)}

Los años 70 y 80 sitúan la emergencia de los estudios sobre el envejecimiento desde las ciencias sociales. Predominaron en este momento visiones generalistas que enmarcaban la vejez como un "problema social" (GIBSON, 1996, p. 434). Aunque se tratan de debates heterogéneos, la mayoría coincidió en poner el foco en el cúmulo de desventajas y carencias que sufrían especialmente las mujeres mayores (GIBSON, 1996, p. 435). Estos estudios identifican una confluencia de situaciones sociales, económicas y políticas que, impactando directamente el bienestar de las mujeres de la tercera edad, las empujan a la experiencia articulada de diversas problemáticas, en estrecha relación con las brechas de género. Estos debates desarrollados avanzan decisivamente hacia la incorporación de interpretaciones multifactoriales e interdisciplinarias sobre la vejez, representando, en este momento histórico específico, un desenlace vanguardista. Cuatro líneas de estudios se desarrollaron en este periodo.

La primera refiere a la formulación en la sociología de un campo de estudios desde el cual se comprende al envejecimiento como parte integral de un proceso histórico-colectivo y a la vez personal: el "curso de vida" ['life course'] (Dale DANNEFER, 201 1, p. 4). Según Leonard Cain (1964) y Glen Elder (1975), este campo de estudios indaga sobre el papel de las circunstancias y eventos concretos vividos por los sujetos o grupos sociales en la forma como envejecen. Sus cuestionamientos se estructuraron alrededor de otros interrogantes complementarios, que observan las diferencias

\footnotetext{
${ }^{2}$ El derecho a jubilarse no está salvaguardado en diversas de las constituciones de los países del sur global. Asimismo, los mercados laborales de estos países contemplan un margen muy expresivo de informalidad, implicando que los y las trabajadoras sean marginados de los beneficios vinculantes de un contrato formal. Incluso en aquellos países donde la legislación reconoce el derecho universal a la jubilación, las pensiones recibidas suelen ser bajas, induciendo los mayores a seguir trabajando.

${ }^{3}$ La impronta crítica de la gerontología se vincula a la función social desempeñada por la disciplina debido a sus finalidades prácticas, dirigidas a solucionar-desde los servicios de atención a la población mayor-problemas sociales acuciantes (OSORIO, 2006, p.6).
} 
del proceso de envejecimiento entre varias generaciones ['cohorts'] (Norman RYDER, 1965; Mathilda RILEY, 1973), las estratificaciones sociales derivadas de la edad (RILEY et al., 1972), y la producción social de la vejez a través de las prácticas cotidianas (Jaber GUBRIUM, 1978). Este campo de estudios incluyó, desde sus inicios, dos orientaciones paradigmáticas (DANNEFER, 201 1, p. 4). 1) La perspectiva "biográfica", enfocada en los patrones y en el desarrollo de eventos rastreables a través de las transformaciones en la trayectoria de los sujetos. 2) El enfoque "institucional", interesado por la organización de estructuras sociales que impactan en la "normalización" del envejecimiento. Desde los 80 (Beth HESS, 1985), estas perspectivas vienen incluyendo una mirada transversal de género (RAMOS-TORO, 2015, p. 14). Pero, como afirman Sara Arber y Jay Ginn (1996, p. 18), los trabajos sociológicos sobre la ancianidad consideraron por décadas al género como una variable, y no como una categoría transversal de la organización social. El cruce sociológico entre edad y género solo aparecería en la sociología en los 90.

La segunda línea vincula preocupaciones sociológicas y antropológicas, indagando sobre la producción social de representaciones que encasillan a las mujeres mayores en un espacio de marginalidad simbólica. Por ejemplo, el estudio de Datan en 1974, en Estados Unidos, sobre la percepción de personas, de diferente edad, género y clase social, sobre la muerte. Según Datan (1989), el estudio observó la preponderancia de una percepción social que denostaba a las mujeres mayores como seres prescindibles. Paralelamente, antropólogas de diferentes orígenes avanzaron en proponer una mirada transcultural que, amparada en la investigación etnográfica en diferentes grupos socioculturales, buscaba contrastar las representaciones simbólicas sobre las mujeres en diferentes momentos de su experiencia vital. Véase, por ejemplo, el libro editado por Michelle Rosaldo et al. (1974).

La tercera de las líneas emerge de la preocupación con las políticas públicas. El trabajo de Carol Estes (1979) fue pionero, examinando la relación entre la política social y las condiciones y necesidades de la población mayor. Concluyó que en esta relación influyen las percepciones, los mitos y los mensajes sobre el envejecimiento que son comunicados tanto a viejos como a jóvenes por los medios y los líderes de opinión, incluyendo a los investigadores gerontológicos (ESTES, 1979, p. 290). Así, las acciones estatales estarían fuertemente impactadas por imaginarios sociales que representan la vejez como un "problema", y por discursos y representaciones que la retratan como una "contrariedad" médica. Por otro lado, también influiría en la relación entre políticas sociales y necesidades de la población en edad avanzada el modo en que la sociedad trata a sus mayores (directa o indirectamente) a través de las políticas sociales de empleo, jubilación, cuidado de salud, ingresos y familia (ESTES, 1979, p. 292). El aporte sustantivo de Estes se distenderá a través de sus estudios durante las décadas de 80 y 90 , evidenciando que las construcciones sociales sobre el envejecimiento desde las políticas sociales reflejan y reproducen disparidades de clase social, género, raza y etnicidad (ESTES, 1993, p. 292). Sus argumentos fueron reiterados por investigadores que, entre 1980 y 1990, abordarían estos temas desde la economía política (Lisa COLEMAN; Sophie WATSON, 1987; HESS, 1985; Sheila PEACE, 1986).

La cuarta de las líneas se vincula al análisis de la relación entre las vulneraciones vividas por las mujeres envejecidas con las responsabilidades "maritales" y familiares relativas a la obligación social del cuidado de hijos, esposos, nietos y padres. Ya en inicios de los 80 , emerge el planteamiento de las desigualdades enfrentadas por las mujeres en relación con los cuidados familiares establecidos hacia parientes en línea ascendiente-de hijas a padres mayores, por ejemplo-, y descendiente-de madres mayores hacia sus hijos y nietos-. En este debate, Elaine Brody (1981) acuñó la frase "mujeres en el medio" para referirse, por un lado, a las mujeres que quedan atrapadas entre las generaciones más vejas y más jóvenes y, por otro lado, a aquellas que combinan las responsabilidades de cuidado parental con el trabajo remunerado (BRODY, 1981). Estos argumentos inaugurarán, como explicaremos adelante, una particular agenda antropológica y sociológica sobre el envejecimiento femenino en las primeras décadas del siglo XXI.

El recurrido sintético por estas cuatro líneas temáticas muestra la expansión del campo de indagaciones e interpretaciones sobre vejez y género. Pero la vinculación crítica entre estos dos elementos solo tuvo lugar en las ciencias sociales a partir de la segunda mitad de los años 80 , y gracias, una vez más, a las investigaciones de la gerontología social (YUNI; URBANO, 2008, p. 152153). Estas preocupaciones serán acompañadas fuertemente por la antropología feminista. En 1985, en el discurso inaugural hecho en la conferencia anual de la Asociación Nacional de Estudios sobre las Mujeres (Estados Unidos), Barbara MacDonald (1989, p.47) reclamaba que: "hasta que no veamos cuán invisibles son las vidas de las mujeres mayores, y por qué lo son, no podemos siquiera empezar el tipo de cambio radical que demanda el desafío del feminismo" (Leni MARSHALL, 2006). Su discurso denunciaba, así, la omisión de la antropología feminista sobre la opresión de la discriminación de las mujeres por edad (MACDONALD, 1989, p. 52). Esta convergencia entre las preocupaciones gerontológicas y antropológicas feminista resultará en un giro epistémico en los estudios sobre mujeres mayores. 


\section{El "giro género-edad" (1990-2000)}

La preocupación de las feministas en los 80 por la fase reproductiva del ciclo de vida dio paso a la conciencia emergente de la post-menopausia, provocando que un pequeño grupo de investigadoras comenzaran a explorar la coincidencia del género y la edad. En los 90 , observamos la emergencia de un debate entre la gerontología social y los estudios feministas en antropología que cuestiona críticamente la interrelación entre género y edad. En el marco de este debate, autoras como Gibson (1996) argumentaron que la edad fuera pasada por alto como un factor estructurante de desigualdades, denunciando que las investigaciones feministas que aplicaban aproximaciones críticas respecto de las divisiones sociales de género, raza y clase, se resistieron a reconocer a la edad como un eje de exclusión. Este giro crítico provocó el surgimiento de un campo analítico específico (YUNI; URBANO, 2008): la antropo-gerontología feminista (TORO-RAMOS, 2015). Uno de los puntos estructurantes de esta vertiente fue el intento de problematizar los estereotipos sociales $-y$ en las ciencias sociales- que estigmatizan a la vejez vinculándola a la decadencia (Thomas COLE et al., 1993):

La gerontología crítica feminista ha documentado la experiencia de las mujeres ancianas promoviendo interpretaciones más completas y complejas acerca de su vida y ha planteado la necesidad de que se estudien y conozcan con mayor detalle sus trayectorias vitales, revisando las lagunas y las inconsistencias que presenta gran parte de la investigación gerontológica actual, víctima de la ideología de la edad. (FREIXAS, 2015, p. 30)

La articulación de este campo interdisciplinar significó la consideración de cuatro características (GIBSON, 1996, p. 442-443): 1) La constatación de que las mujeres mayores experimentan una condensación de problemas y vulneraciones sociales. 2) La crítica sobre el modo como tanto la gerontología social como el feminismo académico analizaron, hasta entonces, la construcción social de las desventajas vividas por las mujeres mayores: dejando de lado en el análisis de las cualidades relacionadas con sus capacidades (por ejemplo, la de construir redes que se mantienen a lo largo del tiempo). 3) Las limitaciones metodológicas de gran parte del trabajo cuantitativo realizado desde la literatura gerontológica, en lo que las distinciones de género y edad se consideraron como variables y no como categorías constructoras de desigualdades. 4) La crítica a la hegemonía epistémica ejercida por los paradigmas positivistas en los estudios de la vejez, visibilizándose la falta de compromiso del feminismo crítico con la investigación de las últimas etapas del ciclo vital, y las insuficiencias de la gerontología al enfocar predominantemente, y por tantas décadas, a la vejez en su relación con la denominada "esfera pública" (jubilación, seguridad salarial).

El debate promovido desde el surgimiento de este nuevo campo se caracteriza por la heterogeneidad. No obstante, hay dos ejes temáticos que quisiéramos recuperar. El primero se refiere a los estudios de la relación entre la feminización de pobreza y el envejecimiento. El clásico trabajo de Robyn Stone (1989) en Estados Unidos inaugura un nuevo eje de debates en este campo. Stone menciona, por un lado, que la longevidad de las mujeres, su tendencia a casarse con hombres mayores que ellas, y las bajas tasas de segundas nupcias entre viudas explican los procesos sociales por los cuales la pobreza se concentra en ellas, mostrando cómo la brecha de género también impacta en esta etapa de la vida. Stone destacó la necesidad de mostrar no sólo las diferencias de género en la vejez, sino también las diferencias raciales. Denunció que el interés de las investigadoras feministas por la pobreza se produjo solamente cuando mujeres blancas de clase media se empobrecieron. Dichos estudios feministas habían visibilizado, así, la necesidad de servicios sociales que atendieran a las mujeres pobres en general, pero sus análisis sublimaron la diversidad de formas con que la pobreza impactaba a las mujeres cuando estas eran negras (STONE, 1989, p.32).

Jacqueline Leavitt y Mary Welch (1989, p. 39) indagarán de su parte sobre las condiciones de vida de las mujeres mayores que viven en los suburbios. Su estudio expuso las dificultades que dichas mujeres enfrentan por tener ingresos más bajos que los hombres, acceso limitado a los automóviles (impidiéndoles movilizarse más cómodamente), y su propensión a vivir solas (condicionándoles a cierto aislamiento). Apuntaron, así, que la inclusión de otras variables analíticas -como la mirada sobre las composiciones familiares, y de las viviendas-, permitían explicar las diversas formas de acceso al bienestar entre las mujeres mayores. Fueron perspicaces al centralizar el compendio de estrategias creativas desarrolladas por estas mujeres para resolver sus necesidades. Con esto, dieron cuenta de un sinfín de actividades económicas informales realizadas por las mujeres mayores y que constituyen emprendimientos económicos centrales para la vida familiar (LEAVITT; WELCH, 1989, p. 40). Observan, además, que estas mujeres invierten mucha energía en la construcción de redes de apoyos (que involucran a familiares, amigos y vecinos). amortiguando así sus dificultades (LEAVITT; WELCH, 1989, p. 39).

Ginn y Arber (1991) revisarían, desde la gerontología crítica, los estudios sociológicos y antropológicos precedentes sobre pobreza. Denuncian que estos reincidían en interpretaciones 
discriminatorias sobre el envejecimiento (padeciendo de Age-ism) y ciegas a la clase social y al género. Su crítica evidenció cómo las concentraciones de pobreza entre las mujeres mayores fueron explicadas en términos de fallas en el sistema de seguridad social, mientras que los factores sociales y económicos que causaban que ellas tuvieran recursos inadecuados simplemente se obviaron. Las autoras comentan que la contribución de las mujeres a la reproducción y la crianza de la próxima generación de empleados, así como las tareas de mantenimiento de quienes se encuentran empleados de hecho, las limita seriamente para asegurar sus propios derechos a pensiones (GINN; ARBER, 1991, p. 392). Mencionan, además, que "el precio de ser mujer" debido a la segregación ocupacional y la subvaloración de su trabajo conectado con "el costo de cuidar" afecta a los ingresos y al bienestar al que acceden cuando ya son mayores (GINN; ARBER, 1991, p. 373-374). Así, estos estudios sobre pobreza e ingresos en la vejez no examinaban en detalle las diferencias de género o no consideraban cómo estas podían estar relacionadas a la experiencia previa a la jubilación, a las limitantes de género en la política social, a las prácticas de contratación y a la asignación de responsabilidades domésticas (GINN; ARBER, 1991, p. 371-372).

Autoras como Judith Gonyea (1994, p. 38-39) acompañarán estas reflexiones mostrando que los programas de política pública orientados a las mujeres mayores en situación de vulnerabilidad debieran aunar la concepción de que el género interpela las diferenciaciones de clase y estatus, a la vez que la constitución etaria puede ecualizar ciertas desventajas para algunas mujeres mayores, mientras para otras las magnifica.

En conjunto, estos trabajos contribuyeron a expandir el concepto de "feminización de la pobreza" señalando los puntos ciegos de las investigaciones precedentes: la insistencia por identificar el género en vez de la clase como el determinante primario de la pobreza y el oscurecimiento de la diferenciación de clase entre las mujeres (GONYEA, 1994, p. 38).

El segundo eje de debates que ganará cuerpo en los 90 desde una perspectiva feminista crítica se vincula a la expansión de los análisis sobre la relación entre cuidados familiares y género influenciados por los debates de Brody (1981). En los 90, varios estudios dialogaron con la expresión de Brody, "mujeres en el medio", buscando entender las encrucijadas femeninas en relación con las responsabilidades del cuidado social. Por ejemplo, Stephen Post (1990) afirmaría que las mujeres toman como "cruciales" las obligaciones de cuidado hacia los padres. No obstante, enmarca esta obligación en sistemas de prestación total que involucran reciprocidades por afinidad. Critica, con ello, los debates de Jane English (1979), para quien la reciprocidad pertenece sólo al presente, y se acerca a los postulados de Christina Sommers (1986), que considera que la compensación que entregan las hijas a los padres es el producto simbólico-relacional de la gratitud por el cuidado recibido durante la niñez (POST, 1990, p. 87). Post (1990, p. 85) destacaría, además, que la transición demográfica, acompañada de la tendencia masculina a evadir el cuidado directo, podría alterar las actitudes de las mujeres sobre esta construcción moral de las obligaciones filiales.

Por su parte, Sarah Boyd y Judith Treas (1989), destacaron que muchos investigadores habían dirigido una considerable atención a "las mujeres en el medio" del trabajo remunerado y del cuidado informal, asumiendo que el conflicto de roles y la sobrecarga las predisponen al estrés. No obstante, los resultados de sus estudios sugieren que, para las mujeres en esta condición, el empleo en el sector productivo no configuraba el determinante más importante del estrés. Las mujeres indicaban, así, la centralidad de la calidad de la relación entre ellas (como cuidadoras) y los sujetos receptores de sus cuidados como elemento que definía su exposición al estrés. Boyd y Treas (1989) añaden que sus múltiples roles proveen a las mujeres mayores de fuentes de satisfacción, no siendo tácitamente un aumento de cargas. Denuncian, entonces, que, al concentrarse exclusivamente en el estrés de la entrega de cuidados, los investigadores ignoraron la resiliencia y las capacidades de adaptación de las mujeres, enviando un mensaje deprimente a las más jóvenes respecto a su futuro (BOYD; TREAS, 1989, p. 71).

\section{Contribuciones latinoamericanas en castellano (2000-2018)}

Para inicios del siglo XXI, la mirada depositada sobre "el problema de las mujeres mayores" fue cambiando, dando origen a un análisis menos "falocéntrico" que superaba la tendencia a establecer a los hombres como una categoría relacional a partir de la cual se definen experiencias y recursos de las mujeres mayores (GIBSON, 1996, p. 433). Este análisis implicó visibilizar ciertas "áreas de silencio": aquellos temas sobre el envejecimiento femenino que no habían sido considerados. Entre ellos, la mayor experiencia de las mujeres mayores en la gestión de la vida en la esfera privada, su involucramiento en la economía informal y su experiencia en el movimiento entre sectores formales e informales (y entre "lo privado" y "lo público") (GIBSON, 1996, p. 436). Esto empujó la perspectiva feminista sobre el envejecimiento a adoptar una mirada crecientemente contextualista:

La investigación debe ir más allá de las suposiciones sobre desigualdades de género universales y más allá de las situaciones desventajadas de las mujeres mayores, para examinar las 
experiencias de los hombres mayores y las mujeres dentro de los contextos en los que ellos viven. (KNODEL; OFTEDAL, 2003, p. 693-694)

En los estudios desarrollados en castellano en América Latina, estas reflexiones impulsaron el redimensionamiento de los estudios sobre el papel de las mujeres con relación a la cadena de reproducción de la vida social dentro, entre y más allá de las familias. Si bien el tema venía siendo levantado desde los 80 , la crítica feminista latinoamericana hispanohablante avanzó a partir de la contribución de investigadoras o bien trabajando en países del sur global, o bien estudiando aquellas mujeres migrantes provenientes de esta región en el norte global. Estos trabajos aúnan esfuerzos de la antropología, sociología y gerontología para plantear la relación concatenada entre los cuidados y las transformaciones que experimentan las mujeres en sus cursos vitales de manera "transgeneracional". Este debate se estructura alrededor de tres conceptos: "cuidados", "trabajos de cuidado" y "organización social de los cuidados".

El concepto de cuidados es polisémico respondiendo a las diversas expresiones cotidianas en las cuales esta práctica puede manifestarse. Alude a los cuidados remunerados y no remunerados (con o sin contrato), que se realizan dentro o fuera de la casa, en un país o entre varios (Herminia GONZÁLVEZ, 2016, p. 45). Todas estas actividades, asignadas cultural e históricamente a las mujeres y naturalizadas en ellas, hacen que la reproducción de la vida sea posible. El problema es cuando esta naturalización desencadena desventajas para las mujeres. El envejecimiento de la población y la feminización de la vejez suelen magnificar estas desventajas, recrudeciendo la sobrecarga femenina en los "trabajos de cuidado" ejercido con parientes ascendientes y descendientes.

Los trabajos de cuidado refieren a tres tipos de actividades entrecruzadas. El cuidado directo dirigido a las personas, el cual incluye cuidado físico (alimentación, baño, aseo), el emocional (escuchar, hablar, ofrecer consuelo) y los servicios para ayudar a la gente a cubrir sus necesidades físicas y emocionales (comprar comida, ir de excursión). El mantenimiento físico de los alrededores en los cuales la gente vive (cambiar la ropa de cama, lavar la ropa, limpiar el suelo). Se relaciona con el fomento de las relaciones y conexiones sociales entre las personas, usualmente conocida como "trabajo de parentesco" (Evelyn GLENN, 2010, p. 5).

Ya la organización social del cuidado es una adaptación regional, latinoamericana, del concepto Social Care propuesto por Mary Daly y Jane Lewis (2000). Alude a la manera como cada sociedad establece una correlación entre sus necesidades de cuidados y la forma como les da respuesta. Es el modo como los actores sociales que pueden tener un papel en la provisión de cuidados (familia, comunidad, mercado y Estado) se combinan y el protagonismo que asumen (Irma ARRIAGADA, 2010).

Los estudios sobre la organización social de los cuidados asumen la centralidad de la vejez, planteando una perspectiva transversal de género. Investiga también a las normas morales (valores y principios laicos y religiosos), las definiciones jurídicas (legislación) y las políticas públicas que determinan en conjunto ( $y$ conflictivamente) qué se entiende por cuidar bien, quién debe cuidar a quién, cómo, dónde, cómo, a cambio de qué. Plantean que la vejez es una etapa del ciclo de la vida cada vez es más duradera -tanto en los países del norte como del sur global-, y desde la que se prolongan responsabilidades más allá de las esperadas. Esto no sólo en cuanto al lugar que ocupan los adultos y adultas mayores como hombres y mujeres, padres, madres, o abuelo/as; sino también por la continuidad de sus responsabilidades en la provisión económica del hogar y en el cuidado de ellos mismos y/o de sus familiares (GONZÁLVEZ, 2017, p. 184). Se plantea que el proceso de envejecimiento es desigual en cuanto al género (Sandra HUENCHUAN, 2010), la clase social (Toni CALASANTI; SLEVIN; KING, 2006) y el parentesco (GONZÁLVEZ, 2017).

Esta perspectiva asume, consecuentemente, un claro posicionamiento político. Se afirma, así, que la de-naturalización de la sobrecarga femenina del cuidado del envejecimiento parte del principio de que este debe ser entendido como un derecho en el marco de las ciudadanías contemporáneas (Jesús GARCÍA, 2009): debe garantizarse por medio de arreglos institucionales y presupuestarios, ser normado y obtener apoyo estatal (Sonia MONTAÑO; Coral CALDERÓN-MAGAÑA, 2010, p. 29).

Parte de las contribuciones específicas de esta perspectiva latinoamericana hispanohablante se refiere a la teorización sobre el efecto acumulativo de la discriminación de género-parentesco y clase social a través del curso de vida, considerando que esto moldea las trayectorias de las mujeres mayores en diferentes contextos regionales. Así, su envejecimiento se constituye desde la condensación de las dificultades y las limitaciones que acumulan progresivamente desde su juventud. Esta condensación se ve claramente reflejada en la degradación de su salud, en el aumento de su dependencia, y en el acceso a jubilaciones/ prestaciones precarizadas, como también concluyen investigaciones realizadas en los países del norte global. A todo esto se le unen dos elementos que, hasta entonces, habían pasado desapercibidos para el análisis de la feminización de la vejez en Latinoamérica. 1) La continuidad del trabajo remunerado más allá de la edad de jubilación tras la aplicación de las reformas 
neoliberales en la región (entre 1980 y 2000), con un incremento de la percepción social de que es deseable no dejar de trabajar. 2) La invisibilidad del continuo trabajo de cuidados (al interior de los hogares y con la familia extensa). Mientras para el trabajo remunerado existe una regulación jurídica que señala cuándo es socialmente posible dejar de hacerlo, los cuidados no cuentan con un paso establecido (ni social ni jurídicamente) hacia la condición de jubilación, especialmente en lo que concierne a las mujeres (OSORIO, 2007, p. 228).

Dado lo anterior, en los sectores urbanos populares de Latinoamérica las mujeres envejecen trabajando tanto dentro como fuera del hogar. Cumplir 60 años no significa dejar de trabajar, ya que continúan apoyando a la familia mediante su trabajo gratuito como cuidadoras informales y también como empleadas domésticas. Muchas mujeres mayores durante su vejez constituyen un significativo sustento de sus núcleos familiares en términos de cuidados materiales, monetarios y emocionales (ONU MUJERES, 2014). Desde estos pilares, se sostiene que el sistema de cuidados en países latinoamericanos es, actualmente, altamente familista (ARRIAGADA, 2010).

\section{Consideraciones finales}

En estas consideraciones finales, nos devolveremos a las dos preguntas que orientaron nuestro estado del arte sobre género, envejecimiento y feminismo.

La primera de ellas indagaba cómo las mujeres mayores fueron categorizadas en las ciencias sociales desde de segunda mitad del siglo XX en adelante. La revisión que presentamos a lo largo del artículo permite establecer que, desde los 70, ha emergido en las ciencias sociales una consciencia de que el envejecimiento es dramáticamente distinto para hombres y para mujeres (DATAN, 1989, p. 15). Se ha sentado un consenso sobre el hecho de que el proceso de envejecer se encuentra impactado por el género-además de otras categorías de diferenciación social como la clase social y la raza. Sin embargo, pese a todos los avances, las necesidades y fortalezas de las mujeres mayores siguen subrepresentadas: tanto en lo que concierne a los estudios sociales, como al discurso hegemónico de las políticas públicas. Sabemos que las mujeres son más eficientes en la mantención de las redes sociales que sustentan la vida, pero también que la pobreza después de los 65 años es principalmente un problema femenino. No obstante, seguimos convocados a hacernos cargo de la heterogeneidad que envuelve la feminización del envejecimiento, no sólo desde los discursos procedentes de la academia, el Estado, o el mercado, sino también desde las propias experiencias de las mujeres.

Desde nuestra perspectiva, esta persistente marginación de las mujeres mayores se refiere al hecho de que ellas siguen constituyendo en los discursos públicos -en la academia y más allá de ella-como vinculadas a una situación de dependencia, desventaja y devaluación. Así, una de las conclusiones de nuestro estado del arte se refiere, precisamente, al hecho de que las mujeres mayores configuran, persistentemente, un "sujeto subalterno" para el pensamiento social. En este sentido, adherimos al debate de Gayatri Chakravorty-Spivak (1998, p.15) para quien los sujetos subalternos se definen por ocupar una posición periférica en el sistema simbólico de producción de los discursos sociales hegemónicos, lo que los involucra en un proceso de violencia epistémica ${ }^{4}$. Dado que la construcción de la diferenciación entre hombres y mujeres se ejecuta en el capitalismo actual siguiendo a procesos de dominio masculino de larga duración (CHAKRAVORTY-SPIVAK, 1998, p. 20), el género femenino es empujado hacia una condición universalizada de subalternidad.

La construcción práctica y simbólica de este lugar de subalternidad femenino, como reflejan los trabajos que revisamos, dista mucho de ser homogéneo (CHAKRAVORTY-SPIVAK, 1998, p. 16). Las mujeres tendrán su marginación magnificada en la medida en que su condición femenina coincide con otros factores delimitadores de la subordinación: la identificación étnica, las etiquetas raciales y sexuales, la condición de clase y, aspecto fundamental para estas consideraciones finales, también su condición generacional. Consideramos que las mujeres mayores representan la condensación de por lo menos dos factores de subalternidad: el género y la edad. Esta subalternidad se conforma de manera tácitamente contradictoria, puesto que estas mujeres ocupan funciones fundamentales -centrales, incluso- para la mantención y reproducción de las sociedades de las que participan y del propio modo productivo. Así, su marginación simbólica reincidente las empuja a la dialéctica condición de ser un "centro silencioso o silenciado", aludiendo, una vez más, a Chakravorty-Spivak (1998, p. 15). Esto implica que sus saberes, prácticas y visiones de mundo han sido marginados en la producción de los discursos sociales hegemónicos: el de las ciencias sociales entre ellos.

En la investigación social contemporánea, la subalternidad de las mujeres mayores está dada, por un lado, por el hecho de que hayan sido invisibilizadas como tema de estudios durante

${ }^{4}$ Esta posición marginada, no obstante, responde a factores estructurales que pueden ser identificados en por lo menos tres escalas. Por un lado, están vinculados a la articulación de mercados, modos de producción y estructuras de poder al interior de los Estados. Pero, por otro lado, también se vinculan con los procesos de colonización que tejen una lógica geopolítica de dominio entre Estados en el sistema mundo. En tercero lugar, la subalternización de los sujetos está interpelada por la forma particular como el capitalismo perpetúa la violencia patriarcal. 
la mayor parte del siglo XX. Por otro lado, también por el hecho de que cuando ellas, al fin, fueron recuperadas -ya en la transición para el siglo XXI-, la mirada analítica predominante interpretaba sus casuísticas vitales definiéndolas, reincidentemente, como un "problema social", o bien enfocándose incisivamente en las "deventajas" de su condición (KNODEL; OFTEDAL, 2003, p. 677).

En este punto, la recuperación que hacemos de Chakravorty-Spivak (1998) coincide con la discusión sobre la "interseccionalidad" promovida por las feministas negras desde los años 90. Según Kimberle Crenshaw (1991, p. 1244), autora que inauguró este debate, las mujeres personifican la superposición de factores excluyentes vinculados a su adscripción étnica, de clase, de edad y generacionales (añadiríamos al argumento de CRENSHAW), que serán mucho más incisivos en su marginación, debido a que compaginan dichas características con su condición de subordinación de género en contextos globalmente patriarcales, machistas y androcéntricos. Esta experiencia de la interseccionalidad define los espacios, derechos y posibilidades de incorporación social femeninos. Así, superando la perspectiva del "doble riesgo", nos gustaría sugerir que el reconocimiento crítico de la condición de subalternidad de las mujeres mayores en la producción del conocimiento social implica una búsqueda por articular diferentes posturas críticas feministas que, cuando entrecruzadas, pueden ayudarnos a remediar la reproducción de ciertos procesos simbólicos (y también materiales) de marginación de las mujeres mayores.

Esto nos lleva directamente a la segunda de las preguntas que estructuraron el desarrollo de este texto: aquella que indaga por la contribución feminista del entendimiento del entroncamiento entre género y envejecimiento.

Terminamos este apartado señalando que, varias décadas más tarde, todavía hay una "deuda del feminismo" con las mujeres mayores (Evelyn ROSENTHAL, 1990). Esta deuda alude a que los estudios sobre estas mujeres son aún un campo marginal dentro de la investigación feminista (Delia GASCÓN, 2009, p. 14), a la vez que se siguen tratando los nuevos estudios sobre el tema como "pioneros", como si los últimos cincuenta años de contribuciones no hubieran sido suficientes para constituir a las mujeres mayores como un sujeto de estudios. Como nos informa Leni Marshall (2006, p. vii), diversas generaciones de escritoras dedicadas a la vejez femenina han sido tratadas por los críticos como las fundadoras del campo. Entre ellas, MacDonald y Cynthia Rich (2001) y Margaret Cruikshank (2003). La pregunta crítica que se hace Marshall (2006, p. vii), hace doce años, invita a una reflexión todavía pertinente en los días actuales: "treinta años después nosotras todavía somos 'pioneras' ¿Cuántas publicaciones se necesitan para pasar a una versión oficial?"

Asimismo, seguimos observando el silencio feminista en materias de políticas relacionadas con personas mayores (GIBSON, 1996, p. 442), sobre todo, en relación con aquellas mujeres para las cuales su situación de desventaja social es impactante (CALASANTI; SLEVIN; KING, 2006, p. 23). Notamos una ausencia de cuestionamiento feminista sobre las mujeres envejecidas, incluso en aquellas áreas de investigación para las cuales el feminismo significó un aporte decisivo, como es el caso del campo de estudios sobre la sexualidad.

La solución de esta deuda implica que avancemos, en el campo de los estudios del envejecimiento, hacia una "perspectiva feminista desde abajo". Se trata de una perspectiva atenta a la contradicción de la figura de autoridad de las investigadoras - reconociendo el lugar de subordinación que ellas propias ocupan en el mundo académico debido a su condición de género (Ruth BEHAR, 1995, p. 2) - y también a los matices políticos del proceso de constitución de las subjetividades entre las personas estudiadas (Kamala VISWESWARAN, 2003, p. 74). Asume que la tensión analítica entre particularismo y universalismos reproduce un ocultamiento de lo femenino como fuente de saber (ROSALDO, 1980). Por lo mismo, se devuelve al carácter parcial y situado del conocimiento científico, buscando con esto

restituir el valor del saber vivencial, implicado y comprometido. Un saber desde el que la razón y la emoción no sean dos polos distinguibles y contrapuestos como el pensamiento occidental androcéntrico y etnocéntrico nos quiere hacer creer, sobredimensionando el valor de lo "racional" frente a lo "emocional", lo científico frente a lo no científico y en el fondo lo masculino, en su asociación aristotélica con la razón, frente a lo femenino en su asociación con lo emocional. (Carmen GREGORIO GIL; Ana ALCÁZAR, 2014, p. 2)

Así, quisiéramos finalizar este artículo proponiendo que el enfoque para los estudios de género y vejez debieran partir de estos principios comunes de la epistemología feminista, pero con el objetivo particular de comprender la dimensión social de "los significados y valores" que condicionan la vida de las mujeres mayores, analizando "las normas culturales que limitan su existencia libre" y también sus "antecedentes y condiciones vitales" (FREIXAS, 2015, p. 28). Simultáneamente, consideramos que teorizar sobre las relaciones de poder basadas en el género y en la edad desde el feminismo implica hablar no sólo de hombres y mujeres, sino también de "los mayores" y el resto de la población. Las personas mayores "sufren desigualdades en la distribución de la autoridad, del estatus y del dinero, y estas desigualdades son vistas como naturales, y, por lo tanto, fuera de disputa" (CALASANTI; SLEVIN; KING, 2006, p. 17-18). 
Los debates latinoamericanos que en la última década han cuestionado estos elementos desde la teorización de los procesos de organización social de los cuidados, constituyen, según entendemos, una contribución fundamental en este sentido.

\section{Agradecimentos}

Agradecemos a la Comisión Nacional de Investigación Científica y Tecnológica (Conicyt) que financia este estudio a través del proyecto Fondecyt Regular 1160683 : "Ser Mujer Mayor en Santiago de Chile: Organización Social de los Cuidados, Feminización del Envejecimiento y Desigualdades acumuladas" (2016-2019), dirigido por Herminia Gonzálvez Torralbo. Agradecemos, además, el trabajo realizado en dicha investigación por el equipo conformado por Franscisca Ortiz, Sofía Larrazábal, Alfonsina Ramírez, Catalina Cano, Javiera Carrasco y Macarena Huaiquimilla.

\section{Referencias}

ARBER, Sara; GINN, Jay. Relación entre género y envejecimiento: enfoque sociológico. Madrid: Narcea, 1996.

ARQUIOLA, Elvira. La vejez a debate: análisis histórico de la situación sociosanitaria de la vejez en la actualidad. Madrid: CSIC, 1995.

ARRIAGADA, Irma. "La crisis de cuidado en Chile". Revista de Ciencias Sociales, Montevideo, v. 23, n. 27, p. 58-67, dic. 2010.

BEHAR, Ruth. "Introduction: Out of Exile". In: BEHAR, Ruth; GORDON, Deborah (Eds.), Women writing culture. Berkeley: University of California Press, 1995. p. 1-33.

BOYD, Sarah; TREAS, Judith. "Family Care of the Frail Elderly: A New Look at 'Women in the Middle'”. Women's Studies Quarterly, Nueva York, v. 17, n. 1/2, p. 66-74, mayo-jul. 1989.

BRODY, Elaine. "Women in the middle and family help to older people". The Gerontologist, Washington, v. 21 , n. 5 , p. $471-489$, oct. 1981 .

BUTLER, Robert. "Age-ism: Another form of bigotry". The Gerontologist, Washington, v. 9, n. 4, p. $243-$ 246, dic. 1969.

CAIN, Leonard. "Life course and social structure". In: FARIS, Robert (Ed.). Handbook of modern sociology. Chicago: Rand-McNally, 1964. p. 271-309.

CALASANTI, Toni; SLEVIN, Kathleen; KING, Neal. "Ageism and Feminism: From 'Eł Cetera' to Center". NWSA Journal, Baltimore, v. 8, n. 1, p. 13-30, abr-sept. 2006.

CHAPPELL, Neena; HAVENS, Betty. "Old and female: Testing the double jeopardy hypothesis". The Sociological Quarterly, Omaha, v. 21, n. 2, p. 157-171, mar. 1980.

CHAKRAVORTY-SPIVAK, Gayatri. “¿Puede hablar el sujeto subalterno?”. Orbis Tertius, La Plata, v. 3, n. 6, p. 1-44, 1998.

COLE, Thomas; ACHENBAUM, Andrew; JAKO $\neg$ BI, Patricia; KASTENBAUM, Robert. Voices and visions of aging. Toward a critical gerontology. Nueva York: Springer, 1993.

COLEMAN, Lisa; WATSON, Sophie. Women over Sixty. Canberra: Australian Institute of Urban Studies, 1987.

CRENSHAW, Kimberle. "Mapping the margins: Intersectionality, identity politics, and violence against women of color". Stanford Law Review, Standford, v. 43, n. 6, p. 1241-1299, jul. 1991.

CRUIKSHANK, Margaret. Learning to Be Old: Gender, Culture, and Aging. Lanham: Rowman, 2003.

CUMMINGS, Elaine; HENRY, William. Growing old. Ann-Arbor: University of Michigan Press, 1961.

DANNEFER, Dale. "Age, the Life Course and the Sociological Imagination: Prospects for Theory". In: BINSTOCK, Robert; GEORGE, Linda (Eds.). Handbook of Aging and the Social Sciences. Londres: Elsevier, 2011. p. 3-16.

DATAN, Nancy. "Aging Women: The Silent Majority". Women's Studies Quarterly, Nueva York, v. 17, n. 1/2, p. 12-19, abril-sept. 1989. 
DALY, Mary; LEWIS, Jane. "The concept of social care and the analysis of contemporary welfare states". British Journal of Sociology, Londres, v. 51, n. 2, p. 291-298, dic. 2000.

DE BEAUVOIR, Simone. La Vejez. Barcelona: Edhasa, 1983[1970].

DEBERT, Guita Grin. A reinvenção da velhice: socialização e processos de reprivatização do envelhecimento. São Paulo: Edusp, 1999.

ELDER, Glen. "Age differentiation and the life course". Annual Review of Sociology, Chicago, v. 1, n. 1, p. 165-190, ago. 1975.

ENGLISH, Jane. "What do grown children owe their parents?". In: O'NEILL, Onora; RUDDICK, William (Eds.). Having children: philosophical and legal reflections on parenthood: essays. Oxford: Oxford University Press, 1979. p. 533-538.

ECKERT, Cornelia. "A cultura do medo e as tensões do viver a cidade: narrativa e trajetória de velhos moradores de Porto Alegre". In: DE SOUZA MINAYO, Maria Cecília; COIMBRA JR, Carlos (Orgs). Antropologia, saúde e envelhecimento. Rio de Janeiro: Editora Fiocruz, 2002. p. 73-102.

ESTES, Carol. The aging enterprise. San Francisco: Jossey-Bass, 1979.

FREIXAS, Anna. "La vida de las mujeres mayores a la luz de la investigación gerontológica feminista". Revista En Otras Palabras, Bogotá, n. 23, p. 23-47, enero-dic. 2015.

GARCíA, Jesús. Los tiempos del cuidado: el impacto de la dependencia de los mayores en la vida cotidiana de sus cuidadores. Madrid: IMSERSO, 2009.

GASCÓN, Delia. Introducción a la Antropología Social: biologicismo, corporalidad y vejez. Ciudad de México: Universidad Estatal del Vale de Ecatepec, 2009.

GIBSON, Diane. "Broken down by Age and Gender: 'The Problem of Old Women' Redefined". Gender and Society, Canberra, v. 10, n. 4, p. 433-448, ago. 1996.

GINN, Jay; ARBER, Sarah. "Gender, Class and Income Inequalities in Later Life". The British Journal of Sociology, Londres, v. 42, n. 3, p. 369-396, sept. 1991.

GLENN, Evelyn. Forced to care: Coercion and caregiving in America. Cambridge: Harvard University Press, 2010.

GONYEA, Judith. "The Paradox of the Advantaged Elder and the Feminization of Poverty". Social Work, Boston, v. 39, n. 1, p. 35-41, ene. 1994.

GONZÁLVEZ, Herminia. "Los cuidados en la migración transnacional. Una categoría de análisis social y políica". Sur. Revista Internacional de Derechos Humanos, São Paulo, v. 13, n. 24, p. 43-52, ago. 2016.

GONZÁLVEZ, Herminia. "Ser Mujer Mayor en Santiago de Chile: feminización de los cuidados en la vejez y desigualdades acumuladas". In: VERA, Antonieta (Ed.). Malestar social y desigualdades en Chile. Santiago: Ediciones Universidad Alberto Hurtado, 2017. p. 173-194.

GREGORIO GIL, Carmen; ALCÁZAR CAMPOS, Ana. "Trabajo de campo en contextos racializados y sexualizados. Cuando la decolonialidad se inscribe en nuestros cuerpos". Gazeta de Antropología, Granada, v. 30, n. 3, p. 1-16, nov. 2014.

GUBRIUM, Jaber. "Notes on the Social Organization of Senility". Urban Life, Nueva York, v.7, n. 1, p. 2344, abr. 1978.

HESS, Beth. "Aging policies and old women: The hidden agenda". In: ROSSI, Alice (Ed.). Gender and the life course. Nueva York: Polity, 1985. p. 98-111.

HUENCHUAN, Sandra. "Envejecimiento y género: acercamiento a la situación específica de las mujeres mayores en América Latina ya las recomendaciones internacionales". In: EQUIPO NIEVE (Comps.). Envejecimiento, género y políticas públicas. Montevideo: UDELAR, 2010. p. 15-32.

JOHNSON, Malcolm. "La conception de la vieillese dans les théories gérontologiques". Retraite et Société, Paris, n. 34, p. 51-67, 2001. 
KNODEL, John; OFSTEDAL, Mary Beth. "Gender and Aging in the Developing World: Where Are the Men?". Population and Development Review, Nueva York, v. 29, n. 4, p. 677-698, dic. 2003.

LEAVITT, Jacqueline; WELCH, Mary Beth. "Older Women and the Suburbs: A Literature Review". Women's Studies Quarterly, Nueva York, v. 17, n. 1/2, p. 35-47, oct. 1989.

LINS DE BARROS, Myriam Moraes. "Trajetória dos estudos de velhice no Brasil". Sociologia, problemas e práticas, n. 52, p. 109-132, ago-dic. 2006.

MACDONALD, Barbara. "Outside the sisterhood: Ageism in women's studies". Women's Studies Quarterly, Nueva York, v. 17, n. 1/2, p. 6-11, 1989.

MACDONALD, Barbara; RICH, Cynthia. Look me in the eye: Old women, aging and ageism. Denver: Spinsters, 2001.

MARSHALL, Leni. "Aging: A Feminist Issue". NWSA Journal, Baltimore, n. 18, p. vii-xiii., abr-jun. 2006.

MONTAÑO, Sonia; CALDERÓN, Coral. El cuidado en acción: entre el derecho y el trabajo. Santiago: CEPAL, 2010.

ONU Mujeres. La organización social del cuidado: identificación de necesidades y escenarios de cuidado. Santiago: ONU Mujeres, 2014.

OSORIO, Paulina. "La longevidad: más allá de la biología. Aspectos socioculturales". Papeles del CEIC, Bilbao, n. 22, p. 1-28, oct. 2006.

OSORIO, Paulina. "Construcción Social de la Vejez y Expectativas ante la Jubilación en Mujeres Chilenas", Universum, Talca, v. 2, n. 22, p. 194-212, 2007.

PEACE, Sheila. "The forgotten female: Social policy and older women". In: PHILLIPSON, Chris; WALKER, Alan (Eds.). Aldershott Ageing and Social Policy: A Critical Assessment. Hants: Aldershot, 1986. p. 96105.

PEIXOTO, Clarice Ehlers. Envelhecimento e imagem: as fronteiras entre Paris e Rio de Janeiro. São Paulo: Annablume, 2000.

POST, Stephen. "Women and Elderly Parents: Moral Controversy in an Aging Society". Hypatia, A Journal of Feminist Philosophy, Nueva York, v. 5, n. 1, p. 83-89, abr-jun. 1990.

RAMOS-TORO, Mónica. Mujeres mayores: Estudio sobre sus necesidades, contribuciones al desarrollo y participación social. 2015. Tesis doctoral (Antropología Social). Departamento de Antropología Social de la Universidad Autónoma de Madrid. Madrid, España.

RILEY, Mathilda. "Aging and cohort succession: Interpretations and misinterpretations". Public Opinion Quarterly, Oxford, v. 37, n. 1, p. 35-47, ene. 1973.

RILEY, Mathilda; JOHNSON, Marilyn; FONER, Anne. Aging and society. A sociology of age stratification. Nueva York: Sage, 1972.

ROSALDO, Michelle. "The use and abuse of anthropology: reflections on feminism and cross-cultural understanding". Signs: Journal of Women in Culture and Society, Chicago, v. 5, n. 3, p. 389-417, abr-jun. 1980.

ROSALDO, Michelle; LAMPHERE, Louise; BAMBERGER, Joane. Woman, Culture, and Society. Stanford: Stanford University Press, 1974.

ROSENTHAL, Evelyn. "Women and varieties of ageism". In: ROSENTHAL, Evelyn (Ed.). Women, aging and ageism. Nueva York: Harrington Park Press, 1990. p. 1-6.

RYDER, Norman. "The cohort as a concept in the study of social change". American Sociological Review, Washington, n. 30, p. 836-841, 1965.

SOMMERS, Christina. "Filial morality". The Journal of Philosophy, Columbia, v. 83, n. 8, p. 439-456, ago. 1986.

STONE, Robyn. "The Feminization of Poverty among the Elderly". Women's Studies Quarterly, Nueva York, v. 17, n. 1/2, p. 20-34, abr-sept. 1989. 
VISWESWARAN, Kamala. "Defining feminist ethnography". In: LINCOLN, Yvonna; DENZIN, Norman (Eds.). Turning Points in Qualitative Research: Tying Knots in a Handkerchief. Nueva York: Altamira Press, 2003. p. 73-94.

YUNI, José Alberto; URBANO, Claudio. "Envejecimiento y género: perspectivas teóricas y aproximaciones al envejecimiento femenino". Revista Argentina de Sociología, Buenos Aires, v. 6, n. 10, p. 151-169, 2008.

Herminia Gonzálvez Torralbo (herminiagonzalvez@gmail.com) es Doctora en Antropología Social y Diversidad Cultural por la Universidad de Granada; Magíster en Migración, Refugio y Relaciones Intercomunitarias por la Universidad Autónoma de Madrid; Licenciada en Antropología Social por la Universidad Miguel Hernández, y Diplomada en Trabajo Social por la Universidad de Alicante. Es investigadora de la Vicerrectoría Académica de la Universidad Central de Chile.

Menara Lube Guizardi (menaraguizardi@yahoo.com.br) es Doctora en Antropología Social y Máster en Estudios Latinoamericanos por la Universidad Autónoma de Madrid (España). Cientista Social y posgraduada en Ciencias Humanas y Desarrollo Regional por la Universidade Federal do Espírito Santo (Brasil). Es investigadora asociada de la Universidad de Tarapacá (Chile) y postdoctorante del Consejo Nacional de Investigaciones Científicas y Tecnológicas (Argentina).

\section{COMO CITAR ESTE ARTícULO, DE ACUERDO CON LAS NORMAS DE LA REVISTA:}

TORRALBO, Herminia Gonzálvez; GUIZARDI, Menara Lube. "As mulheres e o envelhecimento na pesquisa social (1950-2018)". Revista Estudios Feministas, Florianópolis, v. 28, n. 1, e58497, 2020.

\section{CONTRIBUCIÓN DE AUTORÍA}

Herminia Gonzálvez Torralbo: Concepción, recolección de datos y análisis de datos, redacción.

Menara Lube Guizardi: elaboración del manuscrito, redacción, discusión de los resultados.

\section{FINANCIACIÓN}

El artículo se ha desarrollado en el marco del Proyecto Fondecył Regular $n^{\circ} 1160683$ : "Ser Mujer en Santiago de Chile: organización social de los cuidados, feminización del envejecimiento y desigualdades acumuladas" (2016-2019), financiado por la Comisión Nacional de Investigaciones Científicas y Tecnológicas de Chile (CONICYT).

\section{CONSENTIMIENTO DE USO DE IMAGEM}

No se aplica.

\section{APROBACIÓN DE COMITÉ DE ÉTICA EN INVESTIGACIÓN}

El Proyecto Fondecyt Regular $n^{\circ} 1160683$ fue aprobado por el Comité Ético Científico de la Universidad Central de Chile, en consonancia con las especificaciones y requerimientos de la Comisión Nacional de Investigaciones Científicas y Tecnológicas de Chile (CONICYT) y acorde a la normativa establecida por el Fondo Nacional de Desarrollo Científico y Tecnológico de Chile (FONDECYT).

\section{CONFLICTO DE INTERESES}

No se aplica.

\section{LICENCIA DE USO}

Este artículo está licenciado bajo la Licencia Creative Commons CC-BY Internacional. Con esta licencia se puede compartir, adaptar, crear material para cualquier objetivo, siempre que se le atribuya la autoría.

\section{HISTORIAL}

Recibido el 03/08/2018

Presentado nuevamente el 05/04/2019

Aprobado el 29/08/2019 